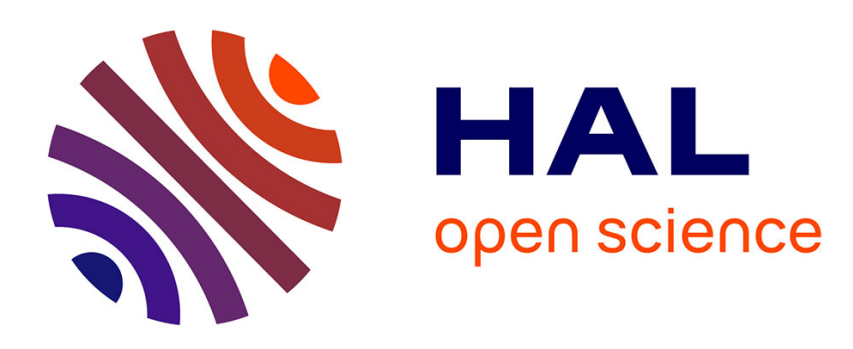

\title{
Sleep: The hebbian reinforcement of the local inhibitory synapses
}

Claude Touzet

\section{To cite this version:}

Claude Touzet. Sleep: The hebbian reinforcement of the local inhibitory synapses. Medical Hypotheses, 2015, 10.1016/j.mehy.2015.06.013 . hal-01337977

\section{HAL Id: hal-01337977 https://hal-amu.archives-ouvertes.fr/hal-01337977}

Submitted on 28 Jun 2016

HAL is a multi-disciplinary open access archive for the deposit and dissemination of scientific research documents, whether they are published or not. The documents may come from teaching and research institutions in France or abroad, or from public or private research centers.
L'archive ouverte pluridisciplinaire $\mathbf{H A L}$, est destinée au dépôt et à la diffusion de documents scientifiques de niveau recherche, publiés ou non, émanant des établissements d'enseignement et de recherche français ou étrangers, des laboratoires publics ou privés. 


\section{Sleep: the hebbian reinforcement of the local inhibitory synapses}

\section{Dr. Claude Touzet}

Integrative and Adaptive Neurosciences (UMR CNRS 7260), Aix-Marseille Univ, 13331 Marseille, France.

Address for correspondence:

Claude Touzet, NIA UMR 7260, Case B, Pôle 3C, Faculté Saint Charles, Aix-Marseille Univeristé, 13331 Marseille, France - Mobile: +33782910319 - Email: claude.touzet@univ-amu.fr

Acknowledgement:

Part of this research was funded by the French Research Agency ANR 2010-CORD-013 "Cognilego - From pixels to semantics: a cognitive approach". We greatly thank Martin Meneval for proofreading the manuscript. 


\begin{abstract}
Sleep is ubiquitous among the animal realm, and represents about $30 \%$ of our lives. Despite numerous efforts, the reason behind our need for sleep is still unknown. The Theory of neuronal Cognition ( $\mathrm{TnC}$ ) proposes that sleep is the period of time during which the local inhibitory synapses (in particular the cortical ones) are replenished. Indeed, as long as the active brain stays awake, hebbian learning guarantees that efficient inhibitory synapses lose their efficiency - just because they are efficient at avoiding the activation of the targeted neurons. Since hebbian learning is the only known mechanism of synapse modification, it follows that to replenish the inhibitory synapses' efficiency, source and targeted neurons must be activated together. This is achieved by a local depolarisation that may travel (wave). The period of time during which such slow waves are experienced has been named the "slow-wave sleep" (SWS). It is cut into several pieces by shorter periods of paradoxical sleep (REM) which activity resembles that of the awake state. Indeed, SWS - because it only allows local neural activation - decreases the excitatory long distance connections strength. To avoid losing the associations built during the awake state, these long distance activations are played again during the REM sleep. REM and SWS sleeps act together to guarantee that when the subject awakes again, his inhibitory synaptic efficiency is restored and his (excitatory) long distance associations are still there.
\end{abstract}




\section{Introduction}

All creatures with a brain are able to learn - and must sleep. Indeed, sleep evidence has been found in most species studied, including cubomedusan jellyfish [1], tree frogs [2], lizards [3], drosophilas [4,5], zebrafish [6], birds [7], and all mammals [8] (starting with the platypus [9]). Sleep may take curious forms such as for the dolphin [10] whose sleep implies only one hemisphere at a time. This happens because breathing is a voluntary act, and requires an awake state. Therefore the dolphin exhibits a two hours sleep in one hemisphere, one hour of awake state for both hemispheres followed by two hours of sleep in the other hemisphere, all over the twelve hours of night. During their migration, several species of birds fly for several days, and sleep while flying a few seconds at a time [11], or one hemisphere at a time.

Sleep seems ubiquitous in the animal realm, and occurs as soon as the species exhibits learning abilities. Only a few (primitive) species seem to be deprived of sleep [8]. Since sleep puts the subject in a dangerous situation with greatly reduced sensory responsiveness (and therefore increased possibility to be eaten by predators), one could conclude that if the process of natural selection has not banished sleep from the behaviour repertoire [12], then it must be mandatory. It looks like sleep is a necessary component of learning - even an integral part of it. It is the opinion of most sleep researchers [13-18].

The duration of sleep varies between species, and also among members of a same species. Humans sleep an average of 8 hours per day, but a few of us are satisfied with only three hours of sleep, while others demand ten hours [8]. Also, sleep requirements varies with the age. Toddlers sleep more than children, who sleep much longer than elderly people [8]. It is acknowledged that young subjects learn more during the day than elderly ones [19], which emphasizes again the relation between learning and sleeping. Since both actions are intrinsically connected, the explanation of sleep must lay in the learning process.

\section{Learning in the brain}

At a neuronal level, learning obeys the hebbian rule, the only known rule of synapse efficiency modification. It was formulated in 1942 by Donald Hebb [20], confirmed by electrophysiology several decades later [21], and since that time, no other proposal has shown up in order to explain learning at a neuronal level. Hebb's rule states that "neurons who fire together get reinforced", and neurons which do not (fire together) see their connection efficiency diminish.

As stated by the Theory of neuronal Cognition ( $\mathrm{TnC})$, the brain does not process information - but represents it [22]. Therefore, it is most important that world regularities are associated to identifiable sets of neurons. The neural localisation of a world event is an identification per se, and allows to act consequently. Neural localization is obtained thanks to inhibitory connections which limit the number of excited neurons at any given time. The proportion of inhibitory connections in the human brain is about $40 \%$ of all connections [23].

In the case of the human species, $22 \%$ of the neurons belong to the cortex [24]. The (human) cortex is composed of about 160,000 cortical columns [25] (a column is a set of about 100,000 neurons), each column belonging to one of the estimated 160 cortical maps that form the cortex. The columnar architecture of the cortex is genetically determined (FIG. 1), but the fine-tuning of its functioning relies on neuroplasticity [26].

\section{Inhibitory synapse efficiency}

Wakefulness functioning requires inhibition to induce localization of the information at the cost of suppressing neighbour activities (in order to end up with only one locally activated column). The inhibitory connections are plastic and obey hebbian learning [33-39]. Since local inhibitory connections are efficient at suppressing the targeted neurons' activity, these inhibitory connections are never reinforced, and their strength diminishes as the day progresses. 
On the contrary, excitatory long distance connections linking two or more columns belonging to separated cortical maps are reinforced as soon as they are activated in the same time frame (Hebb rule again) and are constitutive of the learned experience. As advocated by the TnC, such long distance excitatory connections are representative of « high level » information extraction, and may even account for our flashes of « intelligence » [40].

There is no secret that as the day progresses, we are less and less cognitively efficient. In case of sleep deprivation, the subjects thinking process becomes fuzzy, prone to hallucinations, etc. [41]. Van Dongen et al. [41] provide evidences that the build-up of neurobehavioral deficits is not caused by reduction of sleep time per se, but rather by excessive wakefulness beyond a maximum period during which stable neurobehavioral functioning could be maintained. "Excess wakefulness" is all waking time beyond a hypothetical critical period. They provide results showing a near-linear relationship linking each consecutive hour of wake extension (i.e., excess wakefulness) to an increase in lapses of behavioural alertness (FIG. 2).

Our hypothesis is that the reduced alertness is the result of Hebb learning onto the inhibitory intercolumn connections. Less inhibitory efficiency means that instead of a unique activated column in a given location, there may be two, or more, which induces a less precise representation of the event. If this event is a world situation perceived by the body senses, it may require more time to be recognized (lapse of behavioural alertness). In some cases, it may even be wrongly recognized (confusion, hallucination).

\section{Sleep organization}

After a good (night of) sleep, our brain is again as sharp as usual. Sleep is organized in several stages (II and III) characterized by the fact that the EEG frequency diminishes (from over $20 \mathrm{~Hz}$ to less than $3.5 \mathrm{~Hz}$ ), while the amplitude of the EEG signal becomes several times larger, and paradoxical sleep (also named Random Eye Movements sleep - REM) that resembles the awake state (FIG. 3). It follows that non-REM sleep (NREM) matches sleep stages II and III. More specifically, stage III is also named Slow-Wave Sleep (SWS). It is important to note that surface EEG has been related to underlying neural activity [42] and that the slow waves travel [43].

\section{Hebbian learning balancing}

EEG amplitudes during sleep stages II and III demonstrate that there are several times more neurons acting synchronously than during the wake period. Those neurons are all localized in the same region (beneath the EEG electrode), which means that they belong to neighbouring cortical columns. In particular, the neurons responsible for inter columns inhibitory connections are activated, as are also the neurons targeted by these connections. Following the hebbian learning rule, these inhibitory connections are reinforced. Our hypothesis states that this reinforcement nullifies the previous wakefulness erosions of efficiency.

Our hypothesis also provides an explanation for the succession of various sleeps. The REM sleep is defined by the fact that the EEG is very similar to recordings obtained during the wakefulness period, and also because it follows sleep stages III. It seems that - inserted between periods of inhibitory strength reinforcement - there are periods of long distance excitatory reinforcement (cf. FIG. 4). The explanation may be that during the sleep periods (II and III), the long distance connections are themselves subject to hebbian efficiency erosion (it is the exact inverse situation as in the awake state). Since these connections are important to preserve, they are periodically reinforced during the night.

To resume, limitations of the Hebb's rule impose a balancing between reinforcement of excitatory connections and its concomitant decrease of inhibitory connections (a characteristic of the wake state), and replenishment of inhibitory connection efficiency without impacting the excitatory long distance connections (which is characterizing the sleep). 


\section{Sleep as a localised process}

Recent results show that the quantity of sleep, its repartition among the various stages, as also its local repartition between various zones of the cortex are influenced by the nature and quantity of information acquisition during the day [17]. For example, if today you have done more music exercises than usual, your cortical zones devoted to music will require deeper and longer sleep periods. This is in accord with our hypothesis: it is the inhibitory connection efficiency that regulates the amount and repartition of sleep. Sleep will be displayed by a given cortical zone until the local inhibitory efficiency is restored.

The paradoxical sleep activity has been analysed by Euston et al. [45] as a replay of the day's activations, but not in the same order, and about 6 to 7 times faster. In which case, 90-120 minutes of a night's paradoxical sleep are equivalent to 9-12 hours of wake state. TABLE 1 shows that over the 24 hours day, the NREM sleep exactly compensates for the erosion of the inhibitory efficiency due to wakefulness and REM sleep. It is important to remark that the situation is symmetrical: there is an exact identical compensation of the negative impact of NREM sleep on the excitatory synapse efficiencies. Therefore, in the context of our hypothesis, there is no discrepancy between the duration of both REM and wakefulness compared to NREM sleeps. Replay as also been found during the awake state [46].

\section{The function of sleep}

Nature, with a unique solution available to manipulate the synapse efficiency (Hebb's rule), had to find a trick (using Hebb's rule) in order to replenish the inhibitory efficiency. Since the necessary brain local depolarisations have absolutely no functional relations with the perceived world events, the best solution is to forbid any body movement, any action - which would be necessarily maladapted, even potentially life-threatening. In the context of our hypothesis, sleep is necessarily a period of time during which the body must stay still, whatever the animal species.

\section{Discussion}

Many facts point towards a sleep-dependent memory consolidation. Diekelmann and Born [13] suggest that during slow-wave sleep (SWS), memory representations are transferred from a temporary to a long-term "store" and thereby undergo reorganization in a process of system consolidation. Walker and Stickgold [14] propose to link such processes of reorganization to the REM sleep. Our hypothesis states that the contribution of SWS comes from a better local inhibition, where REM sleep's replay strengthened the long-distance excitatory synapses - both effects allow for a faster and more precise selection of representations.

The synaptic homeostasis hypothesis of Tononi and Cirelli [15] is another proposal for the function of sleep. It hypothesises that plastic processes occurring during wakefulness result in a net increase in synaptic strength in many brain circuits. The role of SWS is then to downscale synaptic strength to a baseline level that is energetically sustainable. The goal of sleep is the homeostatic regulation of the total synaptic weight impinging on neurons. Their hypothesis accounts for a large number of experimental facts, and has implications for both sleep and mood disorders. The synaptic homeostasis hypothesis does not explain the role of REM sleep, otherwise our hypothesis agrees that sleep acts as a homeostatic regulator of the connection strength. Tononi and Cirelli's proposal only focuses on the excitatory connections. Our hypothesis in considering both inhibitory and excitatory synapses could be envisaged as a generalisation of the synaptic homeostasis hypothesis, and explains the role of REM sleep.

Rasch and Born [16] propose that some memory formations require sleep because these formations need to be done "off-line" (due to transient destabilization of memory traces). We do not agree to such proposal which claims that there is a difference in form or nature between the event to record and the long-term memory. In our opinion, the event to record only requires durable synaptic 
strength modifications, and not a transfer to another location. Therefore, there is no requisite for a transient destabilization in order to memorise (since memorisation will only "fix" already experienced pattern of brain activity).

Sejnowski and Destexhe [18] propose that alternating fast (NREM) and slow waves sleep (SWS) consolidate information acquired previously during wakefulness. They propose a plausible biophysical mechanism targeting pyramidal neurons. Our hypothesis differs in the sense that the primary targets of sleep are the inhibitory neurons.

\section{Dreaming}

Dreams fascinate because they do occur without our conscious will, and we do not tolerate to be a puppet in the hands of our neurons. This is the reason why many are searching for dream meaning in the form of premonitory information, or a window into subconscious [47]. In the context of our hypothesis, dreams are less "romantic", they are the results of brain activity reaching language maps, but in a non-coherent manner.

Dreams which occur during NREM sleep (slow wave sleep) are the less numerous [48]. They are the result of the depolarisation waves. These neuronal activities have no link to recent (awake) events. However, there may be recurrent dreams: the same depolarisation waves generating the same cortical columns pattern of activity night after night.

Dreams which occur during the paradoxical sleep (REM) reflect the repeated and disorganised activation of the connections that were once activated during the awake state. It is therefore quite normal to find elements of the previous days, in disorder since there is no order in the replay.

Feelings of flying, weightlessness and impossibility to move seem to be related to the reduction by the reticular active system of the afferent activity (sensors and motor activities) reaching the cortex [49]. We know that we should experience gravity, or that we are sending order to our muscles, but the absence of effect is "logically" interpreted by our brain as an absence of cause: we are floating in the air, we have been tied, etc.

A last word about "premonitory" dreams which occur in particular when the subject is falling asleep, or when he wakes up. They are better built than the other dreams because the brain functioning is less altered at these times. In the light of the TnC, light sleep (LS) affects long distance connections, including those implicated in the verification of the validity of current brain processing representations with our entire life stored events (which requires functional access to the hippocampus, and has a lot in common with hypnosis [50]). These dreams are the result of normal local processing, without supervision (i.e., no comparison to previous experiences). They may provide us with a new (and maybe true) information, an information that (in the awake state) would never have existed because of its lack of coherence with our previous knowledge (a hypothesis similar to Charlton's proposal [51]). Therefore, premonitory dreams exist, but it is our brain that makes the prediction.

\section{Concluding remarks}

Inhibitory neurons amount for a large part of the brain neurons (40\%). They are plastic, a major component of cortical organization, and difficult to study since inhibition effectiveness is ascertained by an absence (suppressed) of activity. More than seventy years after Hebb's insight, we ask and answer the question about how inhibitory synapses could regain their efficiency: "sleep".

Siegel [8] stresses the fact that some animals never exhibit a state that meets the behavioural definition of sleep ("a rapidly reversible state of immobility and greatly reduced sensory responsiveness"). He was not the first one to alert on the limits of this definition. Following the hypothesis we have explored in this paper, a new definition of sleep is necessary. We propose the following: "sleep is when the reinforcement of inhibitory connections - that were lessened by awake functioning - occurs". Sleep may happen in such a way as to impede the quality of the behavioural response. In order to avoid the expression of these maladaptive responses, various 
mechanisms may enter into action such as immobility, reduced sensory responsiveness, etc. Following this new definition, immobility is no longer a prerequisite for sleep, and sleep may occur without immobility. In the same manner, reduced responsiveness may or may not be a side effect of sleep. The question of who sleeps, or doesn't, is not an issue anymore. The right question is "how does a given species succeed to replenish the efficiency of its cortical inhibitory connections?"

Our definition of sleep - the reinforcement of the inhibitory connections - is not readily identified in clinical practice. In order to help promote this new definition, we need to emphasize how it could be implemented, and how it would be applied in the practice of sleep medicine. Today, we know of no tool able to directly measure the strength of the cortical inhibitory connections. But an indirect measure is possible using an (ecologically plausible) test of attention before and after sleeping (TnC states that attention performance is related to the quality of the local cortical inhibition). Also, hebbian learning states that the amount of synaptic strengthening is directly proportional to the number of neuronal co-activations, a number that can be estimated from the amount of delta waves, minus the amount of REM activations. Using the numbers given on table 1 (\% recruitment), it is possible to compute the amount of reinforcements of both excitatory and inhibitory synapses, and therefore estimate the overall quality of sleep, and its components (NREM/REM sleep). Another important factor is the alternation between NREM/REM sleep. A way to evaluate the quality of the alternation is to measure the episodic memory before and after sleep (using interview or selfreport). A degraded performance would be an indicator of a sub-performing REM sleep.

\section{Conflict of interest statement}

The author declares no conflict of interest associated with this publication.

\section{Acknowledgement}

Part of this research was funded by the French Research Agency ANR 2010-CORD-013 "Cognilego - From pixels to semantics: a cognitive approach". We greatly thank Martin Meneval for proofreading the manuscript. 


\section{References}

1. Seymour, J. E., Carrette, T. \& Sutherland, P. Do Box Jellyfish Sleep at Night? Med J Aust., 118:707 (2004).

2. Hobson, J. A., Goin, O. B. \& Goin, C. J. Electrographic Correlates of Behaviour in Tree Frogs, Nature 220, 386-387 (1968). doi:10.1038/220386a0

3. Ayala-Guerrero, F. \& Huitrón-Reséndiz, S. Sleep patterns in the lizard Ctenosaura pectinata, Physiology \& Behavior, 49:6, 1305-1307 (June 1991).

4. Nitz, D.A., van Swinderen, B., Tononi, G. \& Greenspan, R. J. Electrophysiological Correlates of Rest and Activity in Drosophila melanogaster, Current Biology, 12, 1934-1940 (Nov. 19, 2002).

5. Gilestro, G. F., Tononi, G. \& Cirelli, C. Widespread Changes in Synaptic Markers as a Function of Sleep and Wakefulness in Drosophila, Science, 324, 109-112 (2009).

6. Yokogawa, T., Marin, W., Faraco, J., Pézeron, G., Appelbaum, L., Zhang, J., Rosa, F., Mourrain, P. \& Migno, E. Characterization of Sleep in Zebrafish and Insomnia in Hypocretin Receptor Mutants, PLoS Biology 5:10 (October 2007).

7. Amlaner, C. J. \& Ball, N. J. Avian sleep. In Kriger, M.H., Roth, T. \& Dement, W.C. (eds) Principles and Practice of Sleep Medicine, 81-94. W.B. Saunders, Philadelphia (1994).

8. Siegel, J. M. Do all animals sleep? Trends in Neuroscience 31: 208-213 (2008).

9. Siegel, J. M., Manger, P. R., Nienhuis, R., Fahringer, H. M. \& Pettigrew, J. D. Sleep in the platypus. Neuroscience 91(1): 391-400 (1999).

10. Mukhametov, L. M., Supin, A. Y. \& Polyakova, I. G. Interhemispheric asymmetry of the electroencephalographic sleep patterns in dolphins, Brain Research 134(3), 581-584 (14 October 1977).

11. Rattenborg, N.C. Do birds sleep in flight?, Naturwissenschaften. 93(9): 413-25 (September 2006).

12. Cirelli, C. \& Tononi, G. Is sleep essential? PLoS Biol 6(8): (2008) e216. doi:10.1371/journal. Pbio.0060216

13. Diekelmann, S. \& Born, J. The memory function of sleep. Nature Rev. Neurosci. 11, 114-126 (2010).

14. Walker, M. P. \& Stickgold, R. Overnight alchemy: sleep-dependent memory evolution. Nature Rev. Neurosci. 19 Feb 2010 (doi:10.1038/nrn2762-c1).

15. Tononi, G. \& Cirelli, C. Sleep function and synaptic homeostasis. Sleep Med Rev. 10(1):49-62 (Feb 2006).

16. Rasch, B. \& Born, J. Maintaining memories by reactivation. Curr Opin Neurobiol. 17(6):698703 (Dec 2007). doi: 10.1016/j.conb.2007.11.007. Epub 2008 Jan 28.

17.Huber, R., Ghilardi, M. F., Massimini, M. \& Tononi, G. Local sleep and learning. Nature 430(6995):78-81 (2004).

18. Sejnowski, T. \& Destexhe, A. Why do we sleep? Brain Research 886, 208-223 (2000).

19. Ramscar, M., Hendrix, P., Shaoul, C., Milin, P. \& Baayen, H. The Myth of Cognitive Decline: Non-Linear Dynamics of Lifelong Learning, Topics in Cognitive Science 6, 5-42 (2014) doi: $10.1111 /$ tops. 12078

20. Hebb, D. The Organization of Behavior: A Neuropsychological Theory. Wiley (1949).

21. Frégnac, Y., Pananceau, M., René, A., Huguet, N., Marre, O., Levy, M. \& and Shulz, D. A reexamination of Hebbian-covariance rules and spike timing-dependent plasticity in cat visual cortex in vivo, Front. Syn. Neurosci. 2: 147 1-21 (2010).

22. Touzet, C. Consciousness, Intelligence, Free-will: the answers from the Theory of neuronal Cognition - tome 1, Ed. la Machotte, Auriol, F. (in French) (2010).

23. Braitenberg, V. On the Texture of Brains. Heidelberg Science Library (1973).

24. Herculano-Houzel, S. The human brain in numbers: a linearly scaled-up primate brain. Frontiers in human neuroscience, 3:31 (2009).

25. Mountcastle, V. B. The columnar organization of the neocortex, Brain, 120, 701-722 (1997).

26. Verhag, M., Maia, A. S., Plomp, J. J., Brussaard, A. B., Heeroma, J. H., Vermeer, H., Toonen, R. 
F, Hammer, R. E., van den Berg, T. K., Missler, M., Geuze, H. J. \& Südhof, T. C. Synaptic Assembly of the Brain in the Absence of Neurotransmitter Secretion, Science 287, 864 (2000), DOI: $10.1126 /$ science.287.5454.864

27. Kohonen, T. Self-Organizing Maps, 3rd edn. Springer Series in Information Sciences 30 (2001).

28. Buxhoeveden, D. \& Casanova, M. F. The minicolumn hypothesis in neuroscience. Brain 5, 125, 935-95 (2002).

29. Silver, M. \& Kastner, S. Topographic maps in human frontal and parietal cortex. Trends in Cognitive Sciences 13(11), 488-495 (2009).

30. Tanaka, K. Mechanisms of visual object recognition studied in monkeys, Spatial Vision 13, (23), $147-163$ (2000).

31. Favorov, O. V. \& Kelly D. G. Minicolumnar organization within somatosensory cortical segregates. I. Development of afferent connections. Cereb Cortex 4, 408-27 (1994a).

32. Favorov, O. V. \& Kelly D. G. Minicolumnar organization within somatosensory cortical segregates. II. Emergent functional properties. Cereb Cortex 4, 428-42 (1994b).

33. Scelfo, B., Sacchetti, B. \& Strata, P. Learning-related long-term potentiation of inhibitory synapses in the cerebellar cortex, PNAS, 105(2):769-774 (01/2008). DOI:10.1073/ pnas.0706342105

34. Kullmann, D. M., Moreau, A. W., Bakiri, Y. \& Nicholson, E. Plasticity of Inhibition. Neuron 75(6), 951-962 (2012). doi:10.1016/j.neuron.2012.07.030.

35. Maffei, A. The Many Forms and Functions of Long Term Plasticity at GABAergic Synapses. Neural Plasticity (2011) doi:10.1155/2011/254724

36. Gillespie, D. C., Kim, G. \& Kandler, K. Inhibitory synapses in the developing auditory system are glutamatergic, Nature Neuroscience 8, 332 - 338 (2005).

37. Liu, G. Local structural balance and functional interaction of excitatory and inhibitory synapses in hippocampal dendrites, Nature Neuroscience 7, 373 - 379 (2004) doi:10.1038/nn1206

38. Woodin, M. A., Ganguly, K. \& Poo, M. Coincident Pre- and Postsynaptic Activity Modifies GABAergic Synapses by Postsynaptic Changes in Cl\# Transporter Activity, Neuron 39, 807820 (August 28, 2003).

39. Roberts, P. D. System of Mormyrid Electric Fish Modeling Inhibitory Plasticity in the Electrosensory. J Neurophysiol 84:2035-2047, 2000.

40. Touzet, C. The Illusion of Joy. In : J. Schmidhuber, K.R. Thórisson, and M. Looks (Eds.) Artificial General Intelligence 2011, Springer LNAI 6830, 357-362 (2011).

41. Van Dongen, H. P. A., Maislin, G., Mullington, J. M. \& Dinges, D.F. The cumulative cost of additional wakefulness: dose-response effects on neurobehavioral functions and sleep physiology from chronic sleep restriction and total sleep deprivation. Sleep 2:117-126 (2003).

42. Whittingstall, K. \& Logothetis, N. K. Frequency-Band Coupling in Surface EEG Reflects Spiking Activity in Monkey Visual Cortex, Neuron 64(2), 281 - 289 (2009).

43. Massimini, M., Huber, R., Ferrarelli, F., Hill, S. \& Tononi, G. The Sleep Slow Oscillation as a Traveling Wave, The Journal of Neuroscience 24: 6862-6870 (2004).

44. Genzel, L., Kroes, M. C., Dresler, M. \& Battaglia, F.P. Light sleep versus slow wave sleep in memory consolidation: a question of global versus local processes? Trends Neurosci. 37(1): 1019 (2014) .

45. Euston, D., Tatsuno, M. \& McNaughton, B. Fast-Forward Playback of Recent Memory Sequences in Prefrontal Cortex During Sleep, Science 318, 1147 (2007).

46. Carr, M. F, Jadhav, S. P \& Frank, L. M Hippocampal replay in the awake state: a potential substrate for memory consolidation and retrieval, Nature Neuroscience 14, 147-153 (2011)

47. Hobson, J.A. REM sleep and dreaming: towards a theory of protoconsciousness. Nat Rev Neurosci. 10(11):803-13 (2009). doi: 10.1038/nrn2716. Epub 2009 Oct 1.

48. Chellappa SL, Frey S, Knoblauch V, Cajochen C. (2011) Cortical activation patterns herald successful dream recall after NREM and REM sleep. Biol Psychol. 2011 May;87(2):251-6.

49. Evans, B.M. Sleep, consciousness and the spontaneous and evoked electrical activity of the brain. Is there a cortical integrating mechanism? Neurophysiologie clinique 33: 1-10 (2003). 
50. Touzet, C. Hypnosis, Sleep, Placebo: the answers from the Theory of neuronal Cognition tome 2, Ed. la Machotte, Auriol, F. (2014) (in French).

51. Charlton, B.G. \& Andras, P. The Sleep Elaboration-Awake Pruning (SEAP) theory of memory: Long term memories grow in complexity during sleep and undergo selection while awake. Clinical, psychopharmacological and creative implications. Medical Hypotheses, 73(1), 1-4 (2009). 
Fig. 1 a) Following the proposal of T. Kohonen [27], the TnC models the cortex as an homogeneous organization of cortical columns. Each column is a set of about 1,000 minicolumns, each minicolumn containing about 110 neurons (in the human cortex $[25,28]$ ). b) The columns belong to one of the estimated 160 cortical maps, each map is specific to a certain kind of information. The maps belonging to the primary and secondary cortex represent dimensions of the sensory events. Maps of higher level of abstraction combine and fuse information from lower level maps [29]. This fact prohibits an easy interpretation of the information they represent (see Tanaka [30] for examples of high level information representations). The cortical map columns prevent their neighbour activations through lateral inhibition $[31,32]$ - but are potentially excitatorily connected to any other columns of the cortex [23] (adapted from [22]).

Fig. 2 The panels show behavioral alertness, as measured by psychomotor vigilance task (PVT) performance lapses (relative to baseline), plotted as a function of cumulative excess wakefulness (panel A) and as a function of cumulative sleep debt (panel B). Cumulative wake extension (i.e., excess wakefulness - A), rather than cumulative loss of sleep (i.e., sleep debt - B), is the primary cause of progressively reduced behavioural alertness both across days of chronic sleep restriction and across days of total sleep deprivation. Subjects in all four experimental conditions (sleep restriction: $8 \mathrm{~h}$ (diamond), $6 \mathrm{~h}$ (light square), $4 \mathrm{~h}$ (circle) and $0 \mathrm{~h}$ (black square) appeared to experience the same cumulative "cost" (i.e., increase in lapses of behavioural alertness) for each consecutive hour they extended their wake periods (near-linear relationship, fig. A), but if considered from the perspective of sleep debt (fig. B), the response to total sleep deprivation (black square) is fundamentally different from the chronic sleep restriction (adapted from [41]).

Fig. 3 Hypnogram: NREM and REM sleeps alternate in a cyclic fashion. Recordings during the various sleep stages show that wake and REM sleeps EEG are similar, and that NREM sleep involves slower and larger waves (from light sleep to slow wave sleep). A wave is a depolarisation that moves along the cortical tissue. In stage 1 (S1), theta waves appear (frequency between 4-7 Hz and an amplitude between 30 to 50 microvolts). In stage 2 (S2), EEG recordings tend to show characteristic "sleep spindles", which are short bursts of high frequency brain activity (around $13 \mathrm{~Hz}$ ), and "K-complexes" which are delta wave lasting only one second (amplitude between 50 to 100 microvolts). Stage 3 consists of $20 \%$ or more slow waves (delta) with a frequency of less than $1 \mathrm{~Hz}$, and an amplitude around 200 microvolts. Total REM sleep duration is between 90 to 120 minutes, and total sleep is around 8 hours (adapted from [44]).

Fig. 4 During wakefulness (16 hours), the inhibitory connections lose their efficiency. During SWS (dotted line), these connections get reinforced until there are fully replenished at the end of the sleep period (exact same efficiency). During the day, new events are recorded by setting up new long distance excitatory connections (reinforcement of efficiency, we assume a linear progression of the number of new events). During the sleep, the SWS impacts negatively these new connections, but cyclic REM periods (bold line) allow to replay and therefore reinforce these same connections. At the end of the sleep, no excitatory efficiency has been lost. A new day starts with a refreshed inhibition and no excitatory loss (i.e., no memory loss of the previous day's new events). 
Table 1. Duration and percentage of neurons recruited over a 24 hours day

\begin{tabular}{|l|c|c|c|c|}
\hline Effect on synapses & $\begin{array}{c}\text { Reinforcement of excitatory and decrease } \\
\text { of inhibitory connections }\end{array}$ & \multicolumn{2}{c|}{$\begin{array}{c}\text { Reinforcement of inhibitory and decrease } \\
\text { of excitatory connections }\end{array}$} \\
\hline Period of the day & Wakefulness & REM & LS (Light Sleep) & SWS \\
\hline Repartition / day & 16 hours & $1 \mathrm{~h} \mathrm{30}$ & $3 \mathrm{~h} 30$ & $3 \mathrm{~h}$ \\
\hline \multirow{2}{*}{$\begin{array}{c}15 \% \text { recruitment } \\
\text { than REM) }\end{array}$} & $100 \%$ & $50 \%$ & $\begin{array}{c}100 \% \text { (maximal } \\
\text { as in REM) }\end{array}$ \\
\hline $\begin{array}{c}\text { Hours equivalent } \\
100 \% \text { recruiting }\end{array}$ & $2 \mathrm{~h} 24$ & $1 \mathrm{~h} 30$ & $1 \mathrm{~h} 45$ & $3 \mathrm{~h}$ \\
\cline { 2 - 5 } & \multicolumn{2}{|c|}{$4 \mathrm{~h} 54$} & \multicolumn{2}{c|}{$4 \mathrm{~h} 45$} \\
\hline
\end{tabular}




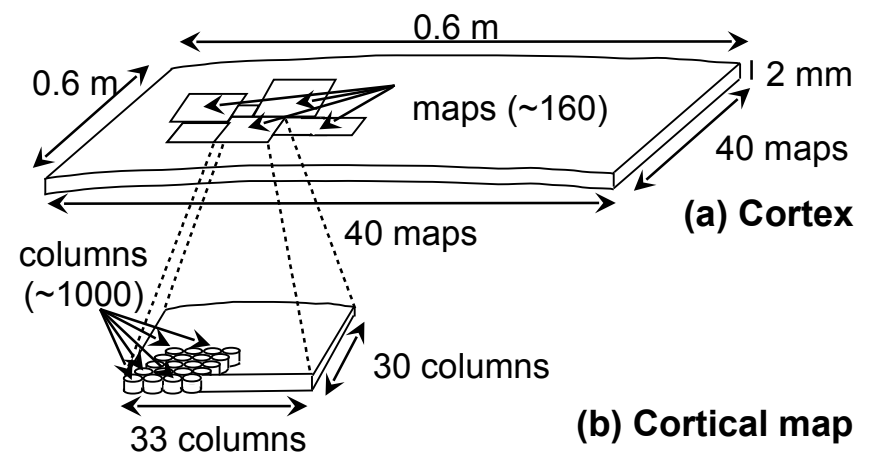

Fig.1 

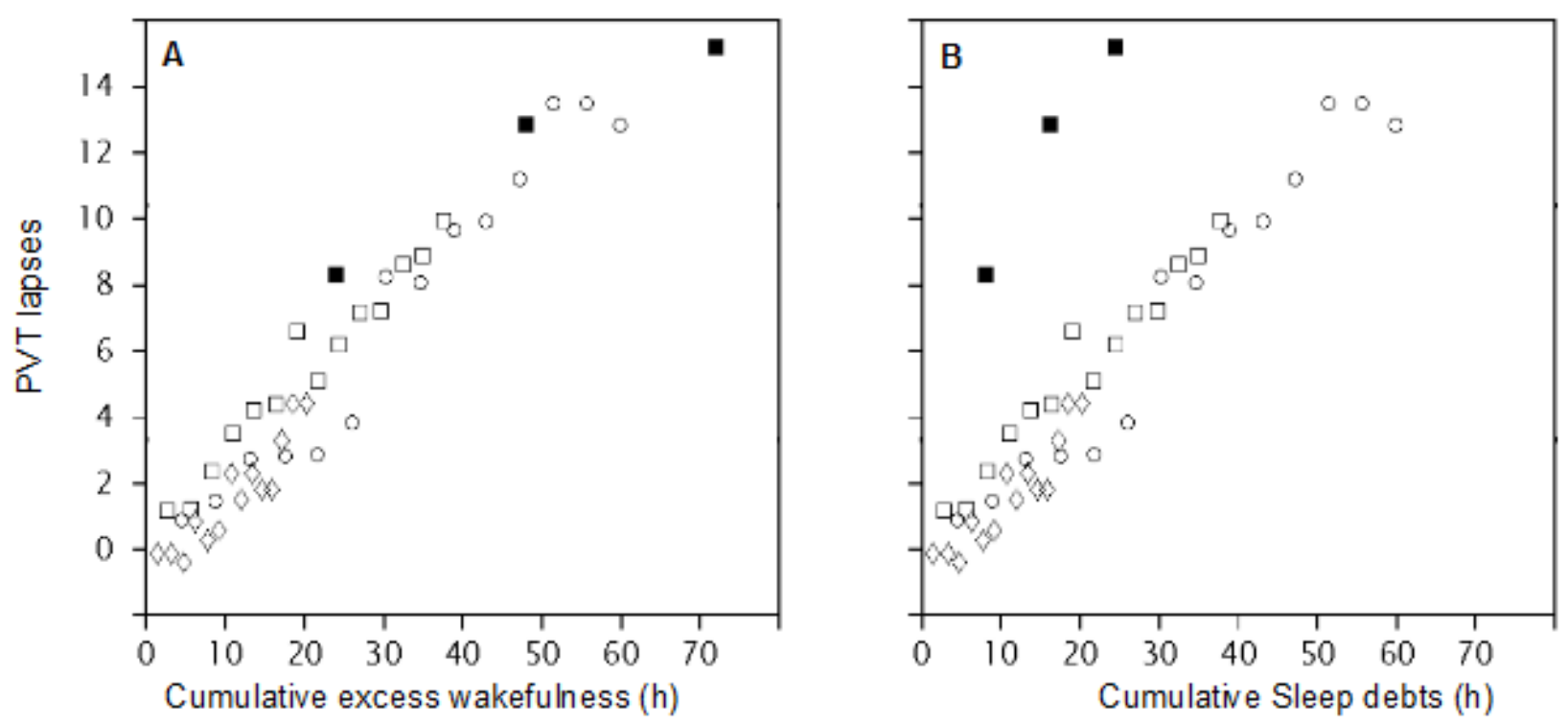

Fig. 2 


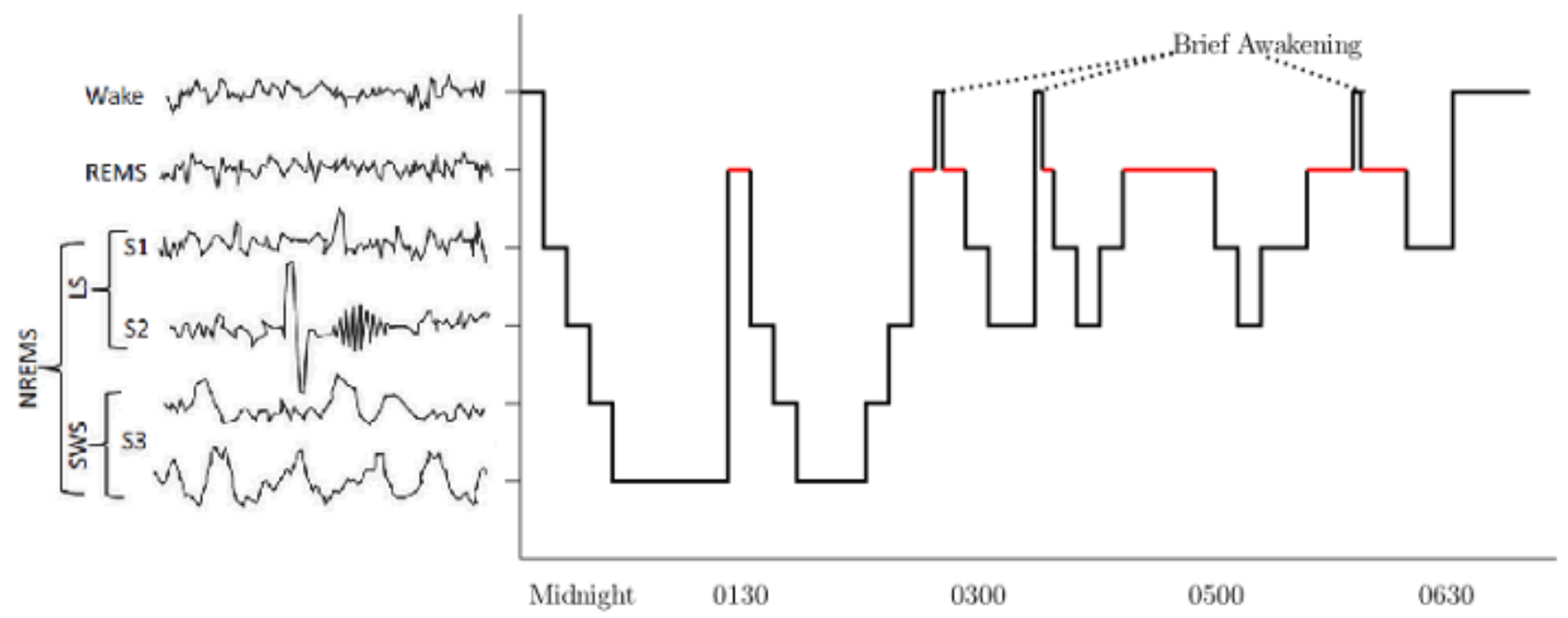

Fig.3 


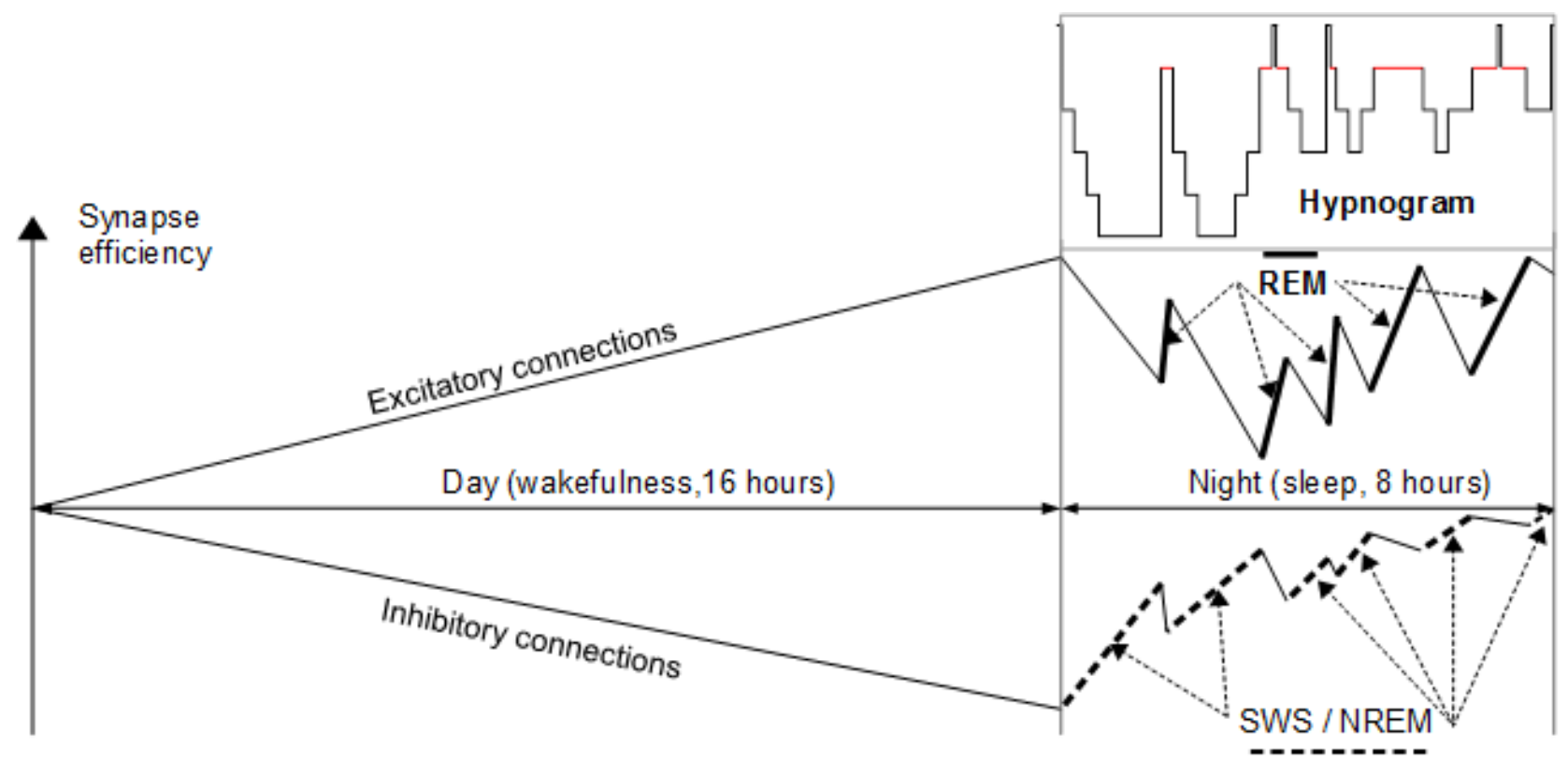

Fig.4 